\title{
Where have the surgical patients gone during the COVID-19 pandemic?
}

\section{Para onde foram os paciente cirúrgicos durante a pandemia do COVID-19?}

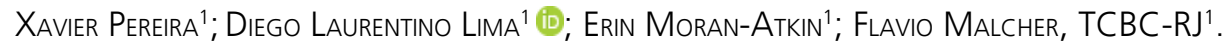

\section{A B S T R A C T}

A Commentary on Emergency Surgery at the Epicenter of the COVID-19 Pandemic.

Keywords: Coronavirus Infections. Pandemics. General Surgery.

\section{MAIN TEXT}

The coronavirus disease 2019 (COVID-19) has caused a significant burden on healthcare systems worldwide. In the United States, New York City quickly became the epicenter of new COVID-19 infections, where the virus disproportionately affected minority communities in high-poverty neighborhoods ${ }^{1}$. In response, our city's hospitals braced for an onslaught of patients, many of whom would require intensive care unit (ICU) level of care. Our institution, which is located in one of the hardest hit zip codes in the country, was quickly forced to restructure and expand inpatient units and to reallocate medical staff in order to deliver highacuity care to over 6,000 patients with COVID-19. Our first COVID-19 case was on March 11, 2020. As our attention shifted to treating those patients affected by the pandemic, surgeons and surgical trainees were called to the frontlines ${ }^{2}$.

As elective surgeries completely ceased on March 18th and our surgical teams consolidated, we remained prepared to care for surgical emergencies and consultations in the same capacity as prior to the pandemic. To our surprise, the volume of surgical emergencies and consultations seemed to drop precipitously in the face of the COVID-19 pandemic. In fact, both of these metrics were inversely proportional to the number of new infections our hospital was treating at any given time during the pandemic (Figure 1).

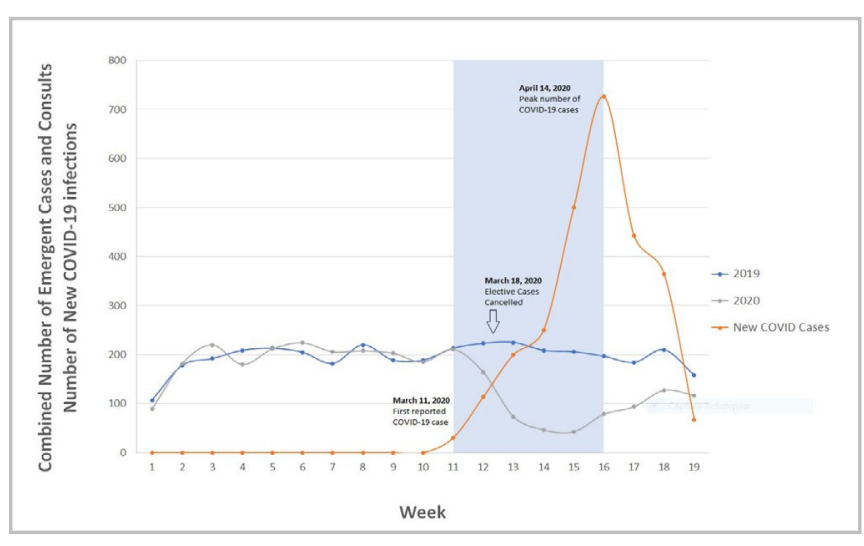

Figure 1. Average combined number of emergency general surgery cases and consultations during the COVID-19 pandemic. Shaded region marks the time between the first confirmed case and the peak number of COVID-19 cases at our institution.

As a high-volume academic center, our institution is uniquely positioned as the primary care provider in the Bronx, NY. Our patient population carries a high burden of complicated surgical disease, which is mediated by a lack of access to adequate care and a high number of comorbid conditions. Each month, there are about 160 general surgery emergency cases and about 640 surgical consultations from inpatient wards or the emergency department. At our hospital, the peak number of COVID-19 positive patients diagnosed in a single day came on April 14, 2020. Since the beginning of the pandemic, our emergency surgical volume has decreased by $52 \%$ when compared to the previous year. The week corresponding to the lowest number of emergency cases was the same week as the highest

1 - Montefiore Medical Center, Department of Surgery - The Bronx - New York - Estados Unidos 
number of new infections, and the period of the highest census of COVID-19 infected patients in the ICU.

The total number of emergency cases completed across our three teaching hospitals during the pandemic was 209, compared to 401 during the same period of $2019(p=0.003)$. This includes a total of 65 appendectomies, 49 cholecystectomies, 2 tracheostomies in the operating room (OR), 48 laparotomies, 9 incarcerated hernia repairs, 13 laparoscopic cases other than appendectomy or cholecystectomy, 2 percutaneous endoscopic gastrostomies (PEG) in the OR, 5 incision and drainage procedures, and 16 operations that we classified as "other".

The number of surgical consultations placed in the inpatient wards and the emergency room followed a nearly identical pattern. During the pandemic, a total of 927 consults were placed to the general surgery service, which compared to 1615 in the same time period last year, corresponding to a $43 \%$ decrease $(p=0.01)$. Interestingly, even though the total volume decreased, the proportion of consultations from the emergency departments remained the same, at about $7.8 \%$ of the total surgical consults placed. Our colleagues in other surgical and medical subspecialties are also reporting reductions in rates of patients with urgent or emergent diagnoses, such as myocardial infarctions and cerebrovascular accidents ${ }^{3,4}$. Patriti et al, a surgical group out of Italy, described a similarly drastic reduction in the need for emergency surgical care ${ }^{5}$.

The decrease in surgical volume is certainly disconcerting and calls into question the notion of what constitutes urgent or emergent surgical cases under normal circumstances. Prior to the pandemic, our institutional practice was to expedite uncomplicated surgical ailments like appendicitis and cholecystitis to the operating room for definitive treatment. These types of cases, which we classified as urgent, were usually brought for operative intervention within 24 hours of admission. This was simply not possible at the peak of the pandemic when our hospital housed about 1,148 COVID-19 patients; making up about $78 \%$ of the total inpatient volume. The surge in positive cases needing hospitalization required a more than $200 \%$ increase in the intensive care unit (ICU) capabilities of our hospital system. Our attending surgeons and surgical residents were tasked with caring for many of these critically ill patients.

It is possible that the reduction in surgical volume was affected by increases in non-operative treatment of common surgical ailments previously considered urgent, such as appendicitis and cholecystitis. While the fear of nosocomial spread of COVID-19 was warranted, without adequate outpatient monitoring and follow-up amidst a pandemic, was that really the safer option? Furthermore, one would expect a surge in emergent and complicated cases of patients who failed non-operative treatment or those who experienced delays in accessing care, but where are these cases? In fact, the number of consultations for these two common ailments was $72 \%$ lower during the pandemic when compared to the same time period in 2019.

Certainly, part of the decrease in volume of consults can be attributed to multiple competing factors. For one, patients may fear coming to hospitals with a high burden of COVID-19 infected patients. They may encounter barriers in getting to the hospital due social distancing recommendations and strained emergency medical response systems. For those who sought care, the overburdened emergency providers may have defaulted to a higher threshold for consulting the surgical services in order to allocate resources to more critically ill COVID-19 patients. The unknown risks of infection transmission during laparoscopy of open surgery meant that surgeons were more likely to entertain more conservative treatments for patients they would have previously operated on. Finally, morbidity and mortality from COVID-19 itself likely acted as a competing risk, by obscuring the observation of surgical emergencies or modifying their chance of occurring.

Patients that sought care during the pandemic seemed to present later in the course of their surgical illness. Data is not yet available comparing the mortality rates of emergency cases during the pandemic to that of previous years. However, we hypothesize that delays in seeking care may have led to increases in the morbidity and mortality. As such, institutions should take into account that an overall reduction of surgical cases may be followed by an increase in severity of surgical disease.

The COVID-19 pandemic challenged our already fragile healthcare system. At our institution, a 
$52 \%$ decrease in general surgery emergencies was seen as a direct result of the pandemic. Other specialties have documented similar decreases in urgent or emergent disease. The impact of delayed surgical care and increased non-operative management of surgical diseases is yet to be evaluated. As several states see new surges in COVID-19 infections it is important for surgical departments to prepare for the accompanying shift in acute surgical needs of the population they serve. It may ultimately allow for better allocation of limited resources.

\section{R E S U M O}

Um comentário sobre cirurgia de emergência em meio à pandemia de COVID 19

Palavras chave: Infecções por Coronavirus. Pandemias. Cirurgia Geral.

\section{REFERENCES}

1. Garg S, Kim L, Whitaker M, O'Halloran A, Cummings $C$, Holstein $R$, et al. Hospitalization Rates and Characteristics of Patients Hospitalized with Laboratory-Confirmed Coronavirus Disease 2019 COVID-NET, 14 States, March 1-30, 2020. MMWR Morb Mortal Wkly Rep. 2020;69(15):458-464. doi:10.15585/mmwr.mm6915e3

2. Nassar AH, Zern NK, Mclntyre LK, Lynge D, Smith $C A$, Petersen RP, et al. Emergency Restructuring of a General Surgery Residency Program During the Coronavirus Disease 2019 Pandemic: The University of Washington Experience. JAMA Surg. 2020;155(7):6247. doi:10.1001/jamasurg.2020.1219
3. Siegler JE, Heslin ME, Thau L, Smith A, Jovin TG. Falling stroke rates during COVID-19 pandemic at a Comprehensive Stroke Center: Cover title: Falling stroke rates during COVID-19. J Stroke Cerebrovasc Dis. 2020;29(8):104953. doi:10.1016/j. jstrokecerebrovasdis.2020.104953

4. Kansagra AP, Goyal MS, Hamilton S, Albers GW. Collateral Effect of Covid-19 on Stroke Evaluation in the United States. N Engl J Med. 2020;383(4):400401. doi:10.1056/NEJMc2014816

5. Patriti A, Eugeni E, Guerra F. What happened to surgical emergencies in the era of COVID-19 outbreak? Considerations of surgeons working in an Italian COVID-19 red zone. Updat Surg. 2020;72(2):309-10. doi:10.1007/s13304-020-00779-6.

\section{Mailing address:}

Diego Laurentino Lima

E-mail: dilaurentino@gmail.com

Conflict of interest: no.

Funding source: none.
Received in: 17/07/2020

Accepted for publication: 18/09/2020 